\title{
EXTENSIONS OF HARDY-LITTLEWOOD INEQUALITIES
}

\author{
ZENGJIAN LOU \\ Mathematics Department \\ Qufu Normal University \\ Qufu Shandong 273165 \\ P. R. China \\ (Received December 30, 1992 and in revised form May 13, 1993)
}

ABSTRACT. For a function $f \in H^{\prime}\left(B_{n}\right)$, with $f(0)=0$, we prove

(1) If $0<p \leqslant s$, then

$$
\int_{0}^{1} r^{-1}\left(\log \frac{1}{r}\right)^{s \beta-1} M_{p}^{s}\left(r, R^{\beta} f\right) d r \leqslant\|f\|_{p}^{s-p}\|f\|_{p, s, \beta}^{p}
$$

(2) If $s \leqslant p<\infty$, then

$$
\|f\|\left\|_{p, s, \beta} \leqslant\right\| f \| p_{p}^{-s} \int_{0}^{1} r^{-1}\left(\log \frac{1}{r}\right)^{s \beta-1} M_{p}^{s}\left(r, R^{\beta} f\right) d r
$$

where $R^{\beta} f$ is the fractional derivative of $f$. These results generalize the known cases $s=2, \beta=$ $1([1])$.

KEY WORDS AND PHRASES. $H^{p}\left(B_{n}\right)$ space, fractional derivative. 1991 AMS SUBJECT CLASSIFICATION CODES. 32A10.

\section{INTRODUCTION.}

Let $C^{n}$ denote the n-dimensional vector space over $C$. Let $B_{n}$ denote the unit ball in $C^{n}$ with boundary $\partial B_{n}$ and let $\sigma$ denote the rotation-invariant positive measure on $\partial B_{n}$ for which $\sigma\left(\partial B_{n}\right)=1$.

We assume that $f$ is holomorphic in $B_{n}$. Let $R^{\beta} f(z)=\sum_{\alpha \geq 0}|\alpha|^{\beta} a_{z} z^{\alpha}$ be the fractional derivative of $f(z)=\sum_{\dot{x} \geq 0} a_{a} z^{*}(\beta>0)$.

For $0<p, s, \beta<\infty$, we set

$$
M_{p}(r, f)=\int_{2 B_{*}}|f(r \zeta)| \cdot d \sigma(\zeta)
$$

and

$$
\|f\|_{p_{,}, \beta, \beta}=\int_{0}^{1} \int_{2 \beta_{*}}|f(r \zeta)|^{\beta-s}\left|R^{\beta} f(r \zeta)\right|^{s}\left(\log \frac{1}{r}\right)^{\beta \beta-1} r^{-1} d \sigma(\zeta) d r
$$

As usual, for $0<p<\infty, H^{p}\left(B_{n}\right)$ denotes the space of holomorphic functions on $B_{n}$ for which the means $M_{p}(r, f)$ are bounded and the norm of $f \in H^{p}\left(B_{n}\right)$ is defined by 


$$
\|f\|_{\rho}=\sup _{0<r<1} M_{p}(r, f) .
$$

Throughout this note, we assume that $f \in H^{P}\left(B_{n}\right)$, with $f(0)=0$.

In [1]. Hardy-Littlewood proved the following well-known theorem about $H^{\prime}\left(B_{1}\right)$.

THEOREM HL. If $0<p \leqslant 2, f \in H^{\prime}\left(B_{1}\right)$, then

$$
\int_{0}^{1}(1-r) M_{p}^{2}\left(r, f^{\prime}\right) d r<\infty
$$

If $2 \leqslant p<\infty$, then $(*)$ implies $f \in H^{\prime}\left(B_{1}\right)$.

In this note, we generalize these results to the unit ball $B_{n}$, with a new and short proof. That is, we prove the following

THEOREM. (1) If $0<p \leqslant s$, then

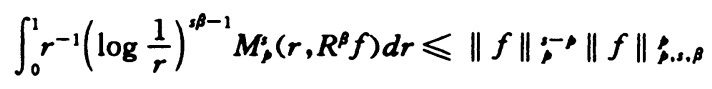

(2) If $s \leqslant p<\infty$, then

$$
\|f\| \beta, s, \beta \leqslant\|f\| \beta^{-s} \int_{0}^{1} r^{-1}\left(\log \frac{1}{r}\right)^{, \beta-1} M_{p}^{\beta}\left(r, R^{\beta} f\right) d r
$$

Set $s=2, \beta=1$ in the Theorem; by the following

LEMMA. For $0<p<\infty$, then

$$
\|f\| p=p^{2}\|f\| p, 2,1
$$

we have the following corollary, which extends Theorem HL (note that for $\zeta \in B_{n}, R^{1} f(\lambda \zeta)=$ $\lambda f^{\prime}{ }_{\xi}(\lambda)$, where $f_{\xi}(\lambda)=f(\lambda \zeta), \lambda \in B_{1}$, and $\left.r \log \frac{1}{r} \sim 1-r\right)$

COROLLARY. (1) If $0<p \leqslant 2$, then

$$
\int_{0}^{1} r^{-1}\left(\log \frac{1}{r}\right) M_{p}^{2}\left(r, R^{1} f\right) d r \leqslant \frac{1}{p^{2}}\|f\|_{p}^{2}
$$

(2) If $2 \leqslant p<\infty$, then

$$
\|f\|_{p}^{2} \leqslant p^{2} \int_{0}^{1} r^{-1}\left(\log \frac{1}{r}\right) M_{\mu}^{2}\left(r, R^{1} f\right) d r
$$

\section{PROOF OF THE MAIN RESULTS.}

PROOF of the Theorem. Let $0<p \leqslant s$. Assume without loss of generality that $\|f\|, \neq 0$.

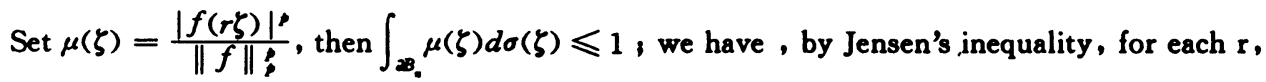

$$
\begin{aligned}
& \left(\int_{\mathbb{B}_{*}}|f(r \zeta)|^{\rho-s}\left|R^{\beta} f(r \zeta)\right|^{\prime} d \sigma(\zeta)\right)^{p / s} \\
& =\left(\|f\| \int_{2 \beta_{1}}\left|\frac{R^{\beta} f(r \zeta)}{f(r \zeta)}\right|^{s} \mu(\zeta) d \sigma(\zeta)\right)^{\alpha / s} \\
& \geqslant\|f\| \rho_{\rho}^{2 / s} \int_{2 \beta_{1}}\left|\frac{R^{\beta} f(r \zeta)}{f(r \zeta)}\right|^{\beta} \mu(\zeta) d \sigma(\zeta) \\
& =\|f\| \beta^{2 / r-p} \int_{\alpha_{0}}\left|R^{\beta} f(r \zeta)\right| \cdot d \sigma(\zeta) \\
& =\|f\| p_{p}^{2 / s-\rho} M_{p}^{\beta}\left(r, R^{\beta} f\right)
\end{aligned}
$$

So

$$
\int_{2 \beta_{+}}|f(r \zeta)|^{p-s}\left|R^{\beta} f(r \zeta)\right|^{s} d \sigma(\zeta) \geqslant\|f\| p^{-s} M_{p}^{s}\left(r, R^{\beta} f\right)
$$


Therefore

$$
\begin{aligned}
\|f\|_{\rho}^{s-p}\|f\|_{p, s, \beta} & =\|f\|_{\rho}^{s-p} \int_{0}^{1} \int_{2 \beta_{0}}|f(r \zeta)|^{\beta-s}\left|R^{\beta} f(r \zeta)\right|^{\prime}\left(\log \frac{1}{r}\right)^{\beta \beta-1} r^{-1} d \sigma(\zeta) d r \\
& \geqslant \int_{0}^{1} r^{-1}\left(\log \frac{1}{r}\right)^{s \beta-1} M_{p},\left(r, R^{\beta} f\right) d r
\end{aligned}
$$

The case $p \geqslant s$ is treated in a similar way to obtain, for each $r$,

$$
\int_{2 B_{.}}|f(r \zeta)|^{p-s}\left|R^{\beta} f(r \zeta)\right|^{s} d \sigma(\zeta) \leqslant\|f\| p^{-s} M_{p}^{s}\left(r, R^{\beta} f\right)
$$

So

$$
\begin{aligned}
\|f\|_{p, s, \beta} & =\int_{0}^{1} \int_{\alpha \beta_{.}}|f(r \zeta)|^{p-s}\left|R^{\beta} f(r \zeta)\right|^{\cdot}\left(\log \frac{1}{r}\right)^{s \beta-1} r^{-1} d \sigma(\zeta) d r \\
& \leqslant\|f\| \rho^{-s} \int_{0}^{1} r^{-1}\left(\log \frac{1}{r}\right)^{s \beta-1} M_{p}^{\prime}\left(r, R^{\beta} f\right) d r
\end{aligned}
$$

This completes the proof of the Theorem.

Now, we use the technique of [2] to give the proof of the lemma.

For $\zeta \in B_{n}, R^{1} f(\lambda \zeta)=\lambda f^{\prime}{ }_{\xi}(\lambda)$, where $f_{\xi}(\lambda)=f(\lambda \zeta), \lambda \in B_{1}$.

By the Hardy - Stein identity for one complex variable $([3])$ we have

$$
\begin{aligned}
M_{p}^{\rho}\left(r, f_{\xi}\right) & =\frac{p^{2}}{2 \pi} \int_{0}^{r} \int_{0}^{2 \pi}\left|f_{\xi}\left(\rho e^{i \theta}\right)\right|^{\rho-2}\left|f_{\xi}^{\prime}\left(\rho e^{i \theta}\right)\right|^{2}\left(\log \frac{r}{\rho}\right) \rho d \theta d \rho \\
& =\frac{p^{2}}{2 \pi} \int_{0}^{r} \int_{0}^{2 \pi}\left|f\left(\rho \zeta e^{i \theta}\right)\right|^{\rho-2}\left|R^{1} f\left(\rho \zeta e^{i \theta}\right)\right|^{2}\left(\log \frac{r}{\rho}\right) \rho^{-1} d \theta d \rho
\end{aligned}
$$

Integrating with respect to $d \sigma(\zeta)$, using the Fubini theorem and the formula

$$
\int_{2 B_{*}} g(\zeta) d \sigma(\zeta)=\frac{1}{2 \pi} \int_{2 B_{*}} d \sigma(\zeta) \int_{0}^{2 \pi} g\left(e^{i \theta \zeta}\right) d \theta, \quad g \in L^{1}(\sigma)
$$

(see $[4, P .15])$, we have

$$
M_{p}^{p}(r, f) \doteq p^{2} \int_{0}^{r} \int_{2 B_{*}}|f(\rho \zeta)|^{p-2}\left|R^{1} f(\rho \zeta)\right|^{2}\left(\log \frac{r}{\rho}\right) \rho^{-1} d \sigma(\zeta) d \rho
$$

Letting $r \rightarrow 1$, we obtain the Lemma.

ACKNOWLEDGEMENT. The author would like to thank the referee for a meticulous reading of the manuscript, in particular for detecting an error in the first version of the Theorem and for several helpful suggestions concerning presentation of the paper.

\section{REFERENCES}

[1] G. H. Hardy and J. E. Littlewood, Notes on the theory of series (XX), Generalizations of a theorem of Paley, Quart. J. Math. , Oxford Ser. , 8(1937), 161-171.

[2] Z. J. Lou, Generalizations of Littlewood and Paley inequalities, Internat. J. Math. \& Math. Sci. , 16(1)(1993), 205-207.

[3] P. Stein, On a theoem of Riesz, J. London Math. Soc. ,8(1933), 242-247

[4] W. Rudin, Function theory in the unit ball of $C^{n}$, Springer-Verlag, New York, 1980. 


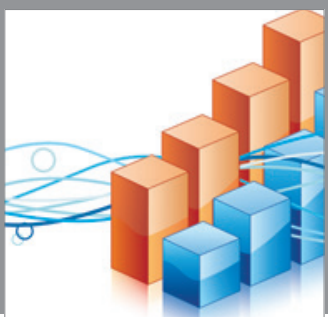

Advances in

Operations Research

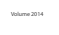

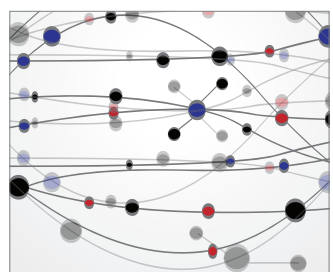

\section{The Scientific} World Journal
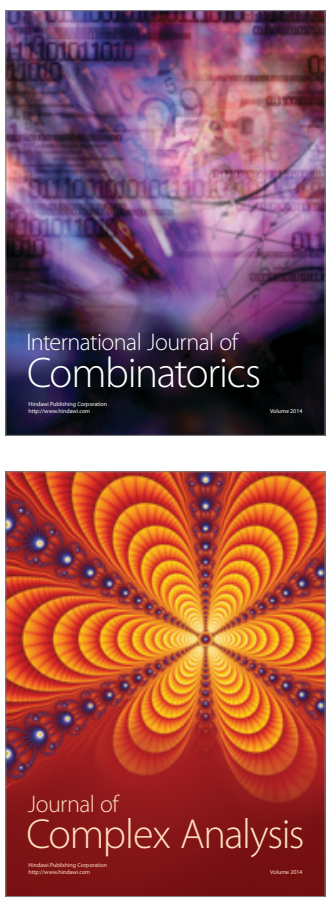

International Journal of

Mathematics and

Mathematical

Sciences
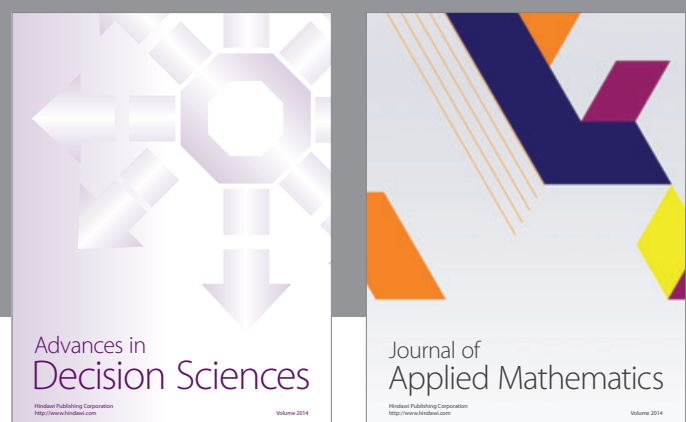

Journal of

Applied Mathematics
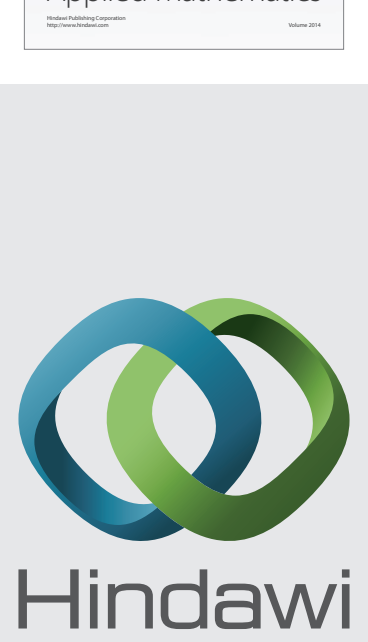

Submit your manuscripts at http://www.hindawi.com
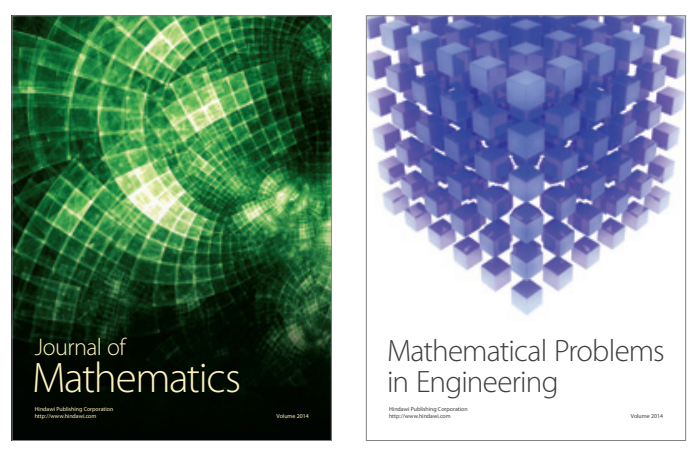

Mathematical Problems in Engineering
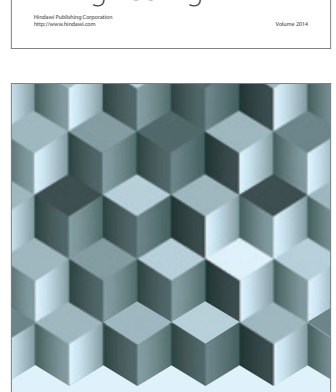

Journal of

Function Spaces
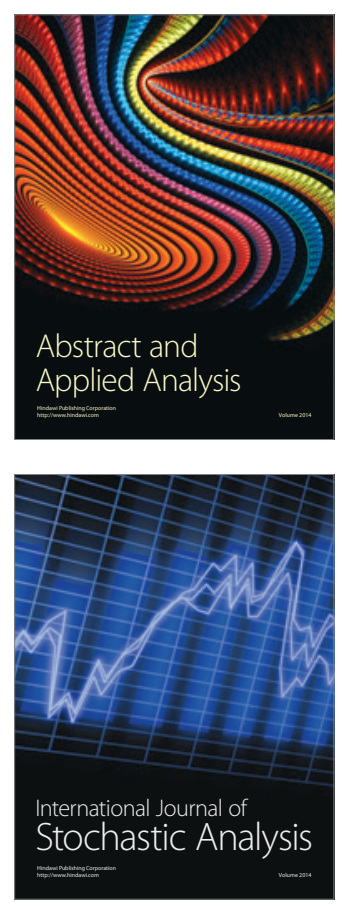

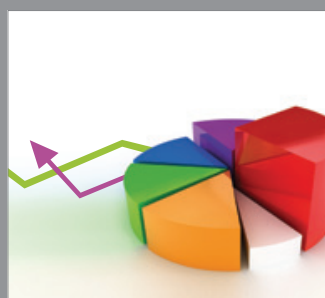

ournal of

Probability and Statistics

Promensencen
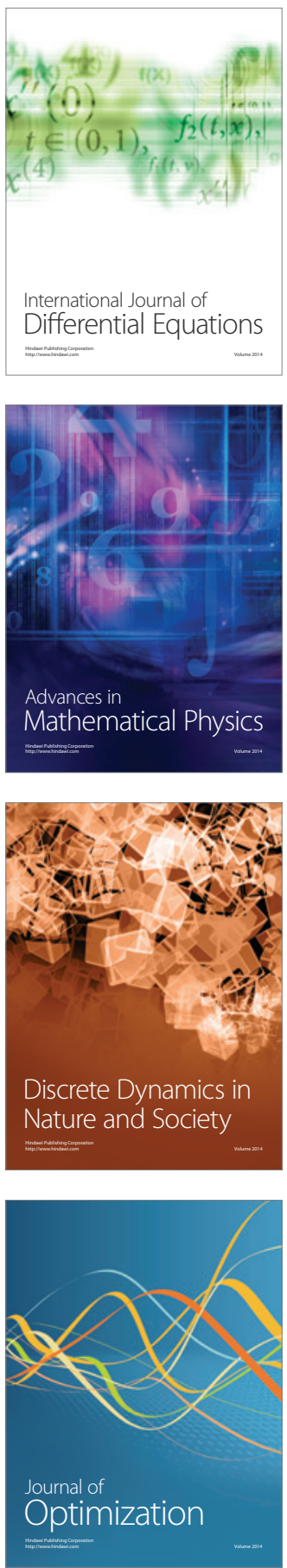